\title{
Simulation of the Solidification of the Melt in the Vanyukov Furnace in the Case of Emergency Stoppage
}

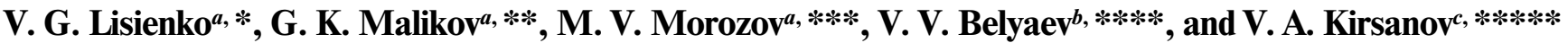 \\ ${ }^{a}$ Ural Federal University, ul. Mira 19, Yekaterinburg, 620002 Russia \\ *e-mail: lisienko@mail.ru \\ **e-mail:german@2-u.ru \\ ***e-mail: liam@mail.utk.ru,mikkael@el.ru \\ ${ }^{b}$ OAO Central Ural Copper Smeltery (SUMZ), ul. Komsomol'skaya 53, Revda, Sverdlovsk Region, 623280 Russia \\ ****e-mail: vvbelyaev@ugmk.com \\ ${ }^{c}$ OOO Ural Mining Metallurgical Company (UGMK), ul. Lenina 1, Verkhnyaya Pyshma, Sverdlovsk Region, 624091 Russia \\ *****e-mail: mvakirsanov@ugmk.ru
}

\begin{abstract}
A mathematical model of the Vanyukov furnace, which makes it possible to predict the behavior of an object in the emergency operational mode (upon the disconnection of the oxygen supply) and develop an effective system of additional heating which damps the consequences of the emergency mode and lowers the costs for the renovation of the furnace operation, is created. It is shown how solidification upon cooling the furnace with time is simulated using the enthalpy and porosity method. The mathematical model is adopted for existing production conditions, which are weakly defined. The energy characteristics of the mode for the solidifying furnace bath, which ensures its holding for a long time in the ready state to rapid firing, are found. Thus, the problem of excessively expensive furnace firing after prolonged production stoppage is solved in the conjugated statement with a calculation of the heating system of the overbath space.
\end{abstract}

Keywords: mathematical modeling, mathematical model, grid model, enthalpy and porosity method, Vanyukov furnace, disconnection of oxygen supply, emergency mode, cooling the melt

DOI: $10.3103 / \mathrm{S} 106782121304010 \mathrm{X}$

\section{INTRODUCTION}

A smelting process performed in a vigorously bubbled matte-slag bath developed under the leadership of Vanyukov (Vanyukov furnace (VF)) is a progressive variant of autogenic processes [1,2]. In this study, we consider processes in the VF as applied to the conditions of the Central Ural Copper Smeltery.

The most dangerous operational mode for the VF is prolonged days-long stoppage, which can take place, for example, in the case of of the breakdown at the oxygen station. In this case there is the probability that the bath will completely freeze and the startup of the aggregate will be expensive. In order to avoid such negative consequences, the bath temperature should be held at the melting point $\left(1200^{\circ} \mathrm{C}\right)$ and the wall accretion on the walls should grow no more than by 150-300 $\mathrm{mm}$.

The heat mode of the bath can be provided using the supply of a definite amount of the gas-air mixture into a furnace space over the wall using the so-called upper heating burners. In this case, flushing the slag below the tuyere level with its loading on the coal surface in order to increase the heat conduction of the surface crust can be used. In order to determine the consumption of natural gas on these upper burners [3], the hydrodynamic and temperature fields for various arrangements of the burners were calculated [3].

To evaluate the growth rate of the wall accretion on caisson-equipped walls, the problem of solidifying the melt in the bath depth should be resolved. The operational practice of using the furnaces shows that the thickness of the wall accretion should not exceed 300 $\mathrm{mm}$ near the gas-oxygen tuyeres; on the contrary, it cannot be broken through, and the operative startup of the furnace becomes impossible. To fulfill this condition, the minimal heat flows should be calculated, which are necessary to hold the wall-accretion thickness at a level of 150-200 mm. The feature of a thus refined simulation of the VF furnace and the goal of this study are to solve both the problems of the heatand-mass exchange, firing, and combustion and the problem of solidifying the slag in a quiet bath.

DESIGN OF A MATHEMATICAL MODEL FOR SOLVING THE SOLIDIFICATION PROBLEM

The solidification and melting of the medium can occur for one temperature (for example, for pure metals) and for the temperature range (for example, in slag 
media). Instead of that, to follow a clear boundary between the solid and liquid phases, it is assumed to use the enthalpy and porosity method [4], which makes it possible to consider the liquid-solid layer in addition to the solid and liquid phases.

\section{Fraction of the Liquid Phase}

In the abovementioned method, the criterion of the separation of the medium into the liquid and solid fractions is specified depending on the temperature and is determined by the limiting levels, between which the linear relationship is accepted.

The numerical characteristic of the fraction of the liquid phase $(\beta)$ shows what the part of the separately taken control volume is in the liquid phase. Depending on the medium temperature,

$$
\beta=\left\{\begin{array}{c}
0, \text { if } T<T_{\text {sol }}, \\
1, \text { if } T>T_{\text {liq }}, \\
\frac{T-T_{\text {sol }},}{T_{\text {liq }}-T_{\text {sol }}}, \text { if } T_{\text {sol }}<T<T_{\text {liq }} .
\end{array}\right.
$$

The magnitude of $\beta$ is calculated at each iteration and for each step in time. It is determined for each control volume in the model; i.e., we can speak about the corresponding field of this parameter and reflect it in a form of the spectral colored pattern similarly to that it is made for the fields of temperatures, velocities, concentrations, etc. The transient liquid-solid zone is considered porous with the porosity coefficient equal to the fraction of the liquid phase.

Equations (1) are suggested by authors [5]. For pure metals, for which the melting and the solidification points are equal, we should apply another approach, which is presented in [4], rather than (1).

In order to take into account the pressure drop caused by the presence of the solid fraction, a corresponding correction member in the pulse equation is foreseen. The corrections are also introduced into the turbulence and enthalpy equations.

Below we present certain main formulas from the theoretical notions which make it possible to obtain the base notion on the numerical simulation of the solidification process. More exact information on the enthalpy method and porosity is given, for example, in [4].

\section{Correction to the Energy Equation}

The enthalpy of the material is calculated as the sum of the visible enthalpy $(h)$ and the latent heat of melting $(\Delta H)$ :

$$
H=h+\Delta H,
$$

where

$$
\begin{gathered}
h=h_{\mathrm{ref}}+\int_{T_{\mathrm{ref}}}^{T} c_{p} d T, \\
\Delta H=\beta L .
\end{gathered}
$$

Here, $h_{\text {ref }}$ is the reference enthalpy, $T_{\text {ref }}$ is the reference temperature, $c_{p}$ is the heat capacity at a constant pressure, $\beta$ is the fraction of the solid phase, and $L$ is the nominal heat of melting of the material.

Thus, the component of the heat of melting can vary from 0 (for the solid phase) to nominal value $L$ (for the liquid phase). The equation of energy for the problems of melting and solidification is written in the operator form similarly to other problems, but enthalpy $H$ is calculated according to (2):

$$
\frac{\partial}{\partial t}(\rho H)+\nabla(\rho \bar{v} H)=\nabla(k \nabla T)+S,
$$

where $\rho$ is the medium density, $\bar{V}$ is the medium velocity, and $S$ is the additive energy source from the reaction model.

It is important to renovate the temperature field at the iteration between the solution of energy equation (5) and the solution of the equation for the part of the liquid fraction (1). The immediate solution of (1) after (5) leads to the unsatisfactory convergence of the problem.

\section{Corrections to the Equations of the Pulse and Turbulence}

The enthalpy and porosity method operates with the transient zone (partially solidified) like with the porous one. The porosity of each control volume is accepted as equal to the value of the part of the liquid fraction. When the material in a certain control volume solidifies completely, the porosity turns to zero and, consequently, velocities also turn to zero. The correction to the pulse in connection with a low porosity in the transient zone takes the following form:

$$
S=\frac{(1-\beta)^{2}}{\left(\beta^{3}+\varepsilon\right)} A\left(\bar{v}-\bar{V}_{p}\right),
$$

where $\varepsilon$ is a small number $(0.001)$ to prevent the possibility of the division by zero in the case when $\beta=0, A$ is the constant of the transient zone, and $\bar{V}_{p}$ is the velocity of the solid phase due to the repulsion of the solidified material from the model space. For our problem, $\bar{V}_{p}$ is accepted to be equal to zero.

In addition, for all turbulence equations in the solid and transient zones, the additive correction is also 
(a)

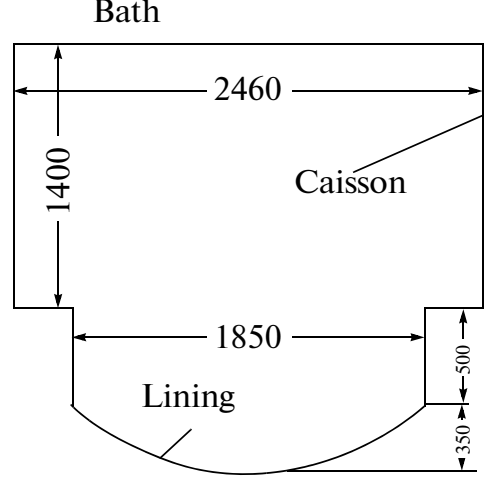

(b)

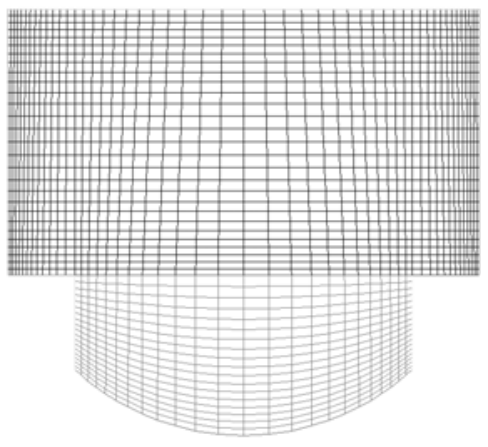

Fig. 1. (a) Computational scheme for the growth simulation of the wall accretion on the VF walls and (b) bulk zones.

introduced. Its definition looks similar to momentum equation (6):

$$
S=\frac{(1-\beta)^{2}}{\left(\beta^{3}+\varepsilon\right)} A \varphi,
$$

where $\varphi$ is the "amount of turbulence," which is obtained from the main turbulence equation according to the accepted model, while the constant of transient zone $A$ is the same constant as in Eq. (6).

Magnitude $A$ in (6) and (7) defines the speed of lowering the medium velocity with its hardening: the higher $A$ is, the more rapidly velocities of the flows lowers to zero upon cooling the material. For most problems, values of $A$ between $10^{4}$ and $10^{7}$ are recommended. Excessively large values can lead to oscillations of the solution (the violation of convergence) since control volumes will alternatively solidify and melt with a small variation in velocity in the zone of the liquid fraction. For the partial problem of cooling the material in the VF, the standard value $A=10^{5}$ was accepted.

To simulate the accretion growth on the VF walls, we constructed an unsteady mathematical model, which makes it possible to investigate the dynamics of the wall-accretion formation in time. For each step in time, the steady problem was solved (the fields of temperatures, velocities, and the fraction of the liquid phase were determined for several iterations).

The computational scheme of this model (Fig. 1a) was constructed using the two-dimensional structured grid. The finest grid is specified near the caisson walls and lining for the detail elaboration of the formation of wall accretion in the first hours after stoppage. In the calculated grid, the surface zones of caissons, lining, and bath surface as well as the bulk zones of the slag and the matte, are distinguished (Fig. 1b).

The properties of the slag and matte accepted to solve the simulation problem are presented in Table 1.

When selecting the densities, we oriented to the data $[2,6]$ presented in rather wide limits, namely, $2800-3700$ and $4700-5700 \mathrm{~kg} / \mathrm{m}^{3}$ for the slag and the matte, respectively. We accepted the average values from the mentioned ranges. We note that the narrower density ranges are presented in other sources, namely, $3500-3700$ and $4700-4900 \mathrm{~kg} / \mathrm{m}^{3}$, respectively.

For calculations, knowledge of the heat conductivity factor was also necessary. To calculate this characteristic, we used its linear dependence on temperature:

$$
\begin{gathered}
\text { for the slag } \lambda=0.003043 t+0.695652 \text {, } \\
\text { for the matte } \lambda=0.007619 t+1.238095 \text {. }
\end{gathered}
$$

For example, we found $\lambda=1.0$ and $2.0 \mathrm{~W} /(\mathrm{m} \mathrm{K})$ for the slag and the matte, respectively. At $t=1250^{\circ} \mathrm{C}$, $\lambda=4.5 \mathrm{~W} /(\mathrm{m} \mathrm{K})$ for the slag and, at $t=1150^{\circ} \mathrm{C}, \lambda=$ $10 \mathrm{~W} /(\mathrm{m} \mathrm{K})$ for the matte.

As for the solidification-melting temperatures, the evaluation of the difference between them is rather complicated if we take into account the very large spread over the compositions of both the slag and the matte. The difference between these temperatures accepted in calculations is approximate and experience shows that it provides a sufficiently acceptable time of iteration calculations using a computer, which considerably increases upon decreasing this difference.

Table 1. Thermal physics properties of the slag and the matte

\begin{tabular}{l|c|c|c|c}
\hline \multicolumn{1}{c|}{ Material } & Heat capacity, $\mathrm{J} / \mathrm{kg}$ & $\begin{array}{c}\text { Latent (hidden) heat } \\
\text { of melting, MJ/kg }\end{array}$ & Density, $\mathrm{kg} / \mathrm{m}^{3}$ & $\begin{array}{c}\text { Solidification } \\
\text { (melting) point, }{ }^{\circ} \mathrm{C}\end{array}$ \\
\hline Slag & 980 & 1.2 & 3000 & $1200(1220)$ \\
Matte & 629 & 0.8 & 5200 & $1100(1120)$ \\
\hline
\end{tabular}


Table 2. Starting data and results of the simulation of the wall-accretion formation for various modes

\begin{tabular}{|c|c|c|c|c|c|}
\hline \multirow{3}{*}{ Variant } & \multicolumn{3}{|c|}{ Starting data } & \multirow{2}{*}{\multicolumn{2}{|c|}{$\begin{array}{c}\text { Results of simulation } \\
\text { thickness of the wall-accretion layer afte } \\
\text { stopping the furnace }\end{array}$}} \\
\hline & \multirow{2}{*}{$\begin{array}{l}\text { specified tempera- } \\
\text { ture of the bath } \\
\text { surface, }{ }^{\circ} \mathrm{C}\end{array}$} & \multirow{2}{*}{$\begin{array}{l}\text { initial temperature } \\
\text { of the slag and matte } \\
\text { zones, }{ }^{\circ} \mathrm{C}\end{array}$} & \multirow{2}{*}{$\begin{array}{l}\text { heat liberation from } \\
\text { chemical reactions, } \\
\mathrm{kW} / \mathrm{m}^{3}\end{array}$} & & \\
\hline & & & & after $22 \mathrm{~h}$ & after $7 \mathrm{~h}$ \\
\hline 1 & 1250 & 1280 & 0 & 280 & 600 \\
\hline 2 & 1250 & 1280 & 0 & 290 & 540 \\
\hline 3 & 1250 & 1280 & 30 & 175 & 150 \\
\hline
\end{tabular}

The following boundary conditions are accepted for all variants of investigation: the temperature of the caisson wall is $100^{\circ} \mathrm{C}$, the temperature of the surrounding medium for the lined walls is $27^{\circ} \mathrm{C}$, and the heat emission coefficient for the lined walls is $0.5 \mathrm{~W} /\left(\mathrm{m}^{2} \mathrm{~K}\right)$.

We considered the cases of the specified (artificially held by heating) temperature of the bath surface of $1250^{\circ} \mathrm{C}$, as well as the variant of the emergency mode of the furnace when there is no heating and the thermal flux by radiation from the bath with a temperature of $1250^{\circ} \mathrm{C}$ on side walls and the arc with a temperature of $100^{\circ} \mathrm{C}$ is specified (emissivity of 0.8 ) (see variant 1 in Table 2).

When modeling, we investigated the influence of heat liberation as a result of the chemical reactions in the slag zone on the dynamics of the wall-accretion formation (this is possible because of the slag properties). In one case we considered a variant with the absence of such reactions, and in the other case, the heat liberation from reactions was $30 \mathrm{~kW} / \mathrm{m}^{3}$. The initial temperature of the slag and matte zones was $1280^{\circ} \mathrm{C}$.

Thickness of the wall-accretion layer, $\mathrm{mm}$

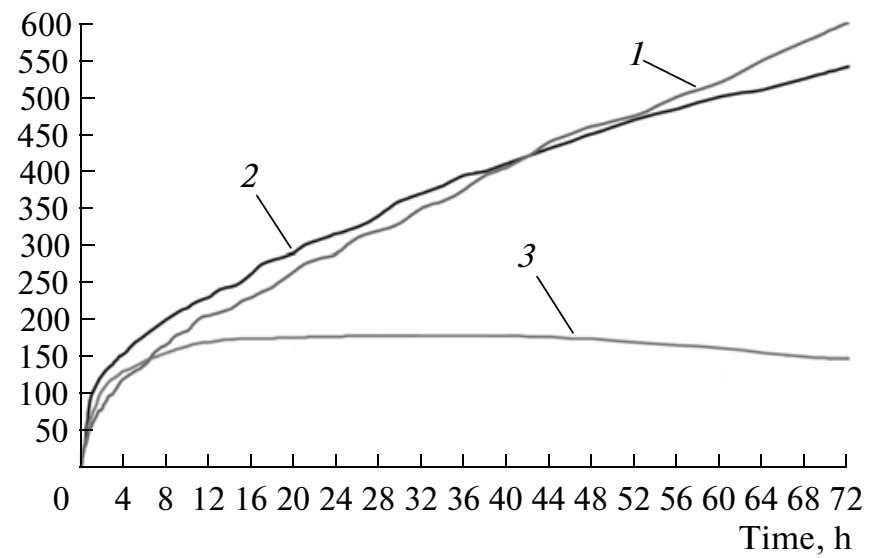

Fig. 2. Dependence of the wall-accretion thickness in the slag zone on time. Digits at the curves are the variant of the mode: (1) without heating the bath surface, (2) with its heating without allowing for the reactions, and (3) with heating and allowing for the reactions.
The mode variants of the experiments and the main results of simulation are tabulated in Table 2 and presented in Figs. 2 and 3.

The density of the heat flows on the walls of the slag zone with a wall-accretion thickness of 150-200 mm is in the limits of $18-13 \mathrm{~kW} / \mathrm{m}^{2}$ (Fig. 3); this made it possible to determine the amount of heat necessary to hold this thickness $\left(18 \mathrm{~kW} / \mathrm{m}^{2}\right)$ for several days: $18 \mathrm{~kW} / \mathrm{m}^{2} \times 42 \mathrm{~m}^{2}=756 \mathrm{~kW}$, where $42 \mathrm{~m}^{2}$ is the surface of the walls of the slag zone. To form this heat flux, we can supply natural gas and compressed air through the oxygen tuyeres. When burning $1 \mathrm{~kg} / \mathrm{s}$ of natural gas (accepting the temperature of exhaust smoke equal to a bath temperature of $1200^{\circ} \mathrm{C}$ ), we can transfer heat of $22 \mathrm{MJ}$ to the bath.

In order to transfer heat of $756 \mathrm{~kW}$ to the bath, a supply of $0.0344 \mathrm{~kg} / \mathrm{s}$ or $170 \mathrm{~m}^{3} / \mathrm{h}$ of the natural gas and, correspondingly, $1700 \mathrm{~m}^{3} / \mathrm{h}$ of the compressed air is necessary.

Since the bath surface is held in the liquid state due to burning (its calculation is given in [3]), bubbles of the exhaust smoke freely escape into the furnace space. In reality, the necessary amount of natural gas

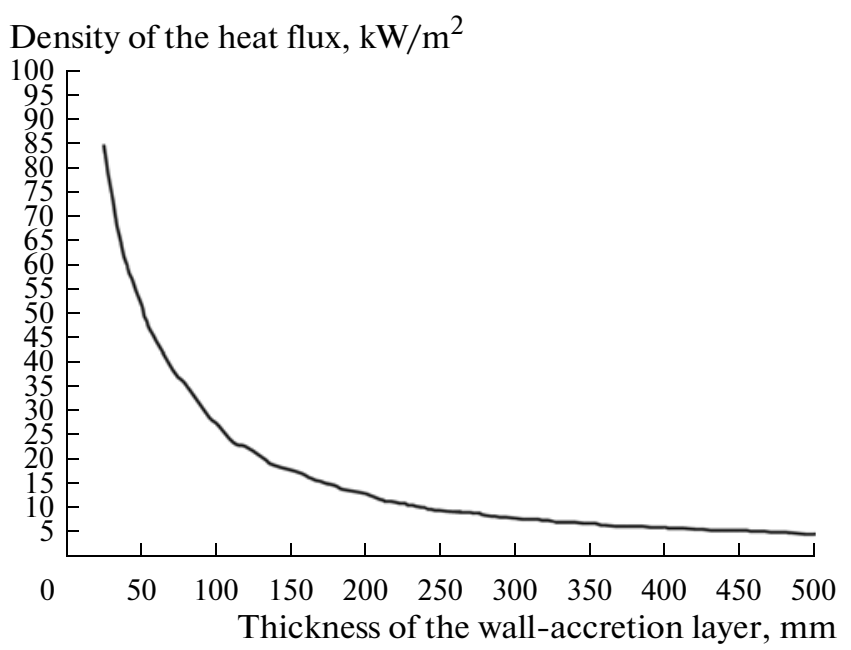

Fig. 3. Dependences of the density of the heat flow to the caissons on the wall-accretion thickness during cooling. 
and compressed air over the bath will be considerably smaller, since bubbling of the liquid part of the slag bath will increase its effective thermal conductivity by a factor of several times and heat from the burning process over the bath will be transferred downward in considerably larger amounts.

\section{RESULTS OF THE SIMULATION OF THE SOLIDIFICATION}

(i) The numerical modeling of "freezing" the bath in the matter and slag zones after the emergency stoppage of the VF is performed. The magnitudes and rates of growth of the wall accretion of the caisson walls (the slag) and the lined walls (the matte) are determined.

(ii) The magnitude of the wall accretion-incrustation on the caisson walls $22 \mathrm{~h}$ after stopping the VF reaches $280-290 \mathrm{~mm}$ in the absence of the chemical reactions in the slag zone and $175 \mathrm{~mm}$ in the case of low-intensity reactions (with heat liberation not exceeding $30 \mathrm{~kW} / \mathrm{m}^{3}$ in the zone of the slag melt).

Two smeltery visual results confirm these conclusions: a wall-accretion thickness of $150-180 \mathrm{~mm}$ is formed for $22 \mathrm{~h}$, and $300-330 \mathrm{~mm}$ is formed for $26 \mathrm{~h}$ after stopping the VF. In the first case, continuing lowintensity reactions in the slag zone act; in the second case, the reactions are absent (this depend on the charge composition).

(iii) The thickness of the wall accretion-incrustation on the walls in the matte zone is negligibly small, and even after 96-h stoppage it does not exceed $5 \mathrm{~mm}$ for the variant without reactions.

(iv) Holding the thickness of the wall accretion $(150-180 \mathrm{~mm})$ for $4-5$ days can be guaranteed by the supply of a small amount of the natural gas $\left(170 \mathrm{~m}^{3} / \mathrm{h}\right)$ and compressed air $\left(1700 \mathrm{~m}^{3} / \mathrm{h}\right)$ through the oxygen tuyeres in the slag zone.

\section{CONCLUSIONS}

The considerable advantages of the VF by some main technical and economical characteristics do not diminish the role of improving systems for controlling the process, especially in view of preventing emergencies and excluding their possible consequences. The modern methods of mathematical modeling makes it possible, even as applied to such a complex aggregate as the VF, to calculate and analyze the radiation and complex heat exchange, hydrodynamics, burning, solidification, and melting. In this study we demonstrated the possibility of a detailed evaluation of the thickness and the growing rate of the wall accretion on the caisson and lined walls in the case of stopping and cooling the furnace because of the disconnection of the oxygen supply. In this case, the recommended consumption of natural gas to upper burners for holding the wall-accretion thickness at a level of $150-180 \mathrm{~mm}$ for 4-5 days is evaluated. The visual smeltery observations and the operational practice of the furnace in general confirm the results of model evaluations.

\section{REFERENCES}

1. Vanyukov, A.V., Bystrov, V.P., Vaskevich, A.D., et al., Plavka v zhidkoi vanne, (Smelting in the Liquid Bath), Vanyukov, A.V., Ed., Moscow: Metallurgiya, 1988.

2. Lisienko, V.G., Shchelokov, Ya.M., and Ladygichev, M.G., Plavil'nye agregaty: teplotekhnika, upravlenie i ekologiya: Spravochnoe izdanie v 4 knigakh. (Smelting Aggregates: Heat Engineering, Control, and Ecology: Hanbook in 4 vols), Lisienko, V.G., Ed., Moscow: Teplotekhnik, 2005, vols. 1-2.

3. Lisienko, V.G., Malikov, G.K., Morozov, M.V., Belyaev, V.V., and Kirsanov, V.A., Rus. J. Non-Fer. Met., 2012, no. 3, p. 272.

4. Voller, V.R. and Prakash, C., Int. J. Heat Mass Transfer, 1987, vol. 30, p. 1709.

5. Voller, V.R. and Swaminathan, C.R., Numer. Heat Transfer B, 1991, vol. 19, no. 2, p. 175.

6. Kobakhidze, V.V., Teplovaya rabota i konstruktsii pechei tsvetnoi metallurgii (Thermal Work and Designs of the Furnaces of the Nonferrous Metallurgy), Moscow: MISiS, 1994.

Translated by N. Korovin 